\title{
ON THE PRIME SUBMODULES OF MULTIPLICATION MODULES
}

\author{
REZA AMERI
}

Received 28 February 2002

\begin{abstract}
By considering the notion of multiplication modules over a commutative ring with identity, first we introduce the notion product of two submodules of such modules. Then we use this notion to characterize the prime submodules of a multiplication module. Finally, we state and prove a version of Nakayama lemma for multiplication modules and find some related basic results.
\end{abstract}

2000 Mathematics Subject Classification: 16D10.

1. Introduction. Let $R$ be a commutative ring with identity and let $M$ be a unitary $R$-module. Then, $M$ is called a multiplication $R$-module provided for each submodule $N$ of $M$; there exists an ideal $I$ of $R$ such that $N=I M$. Note that our definition agrees with that of [1, 2], but in [6] the term multiplication module is used in a different way. (In this paper, an $R$-module $M$ is a multiplication if and only if every submodule of $M$ is a multiplication module in the above sense.) Recently, prime submodules have been studied in a number of papers; for example, see $[3,4,5]$. Now in this paper, first we define the notion of product of two submodules of a multiplication module and then we obtain some related results. In particular, we give some equivalent conditions for prime submodules of multiplication submodules. Finally, we state and prove a version of Nakayama lemma for multiplication modules.

2. Preliminaries. Throughout this paper, $R$ denotes a commutative ring with identity and all related modules are unitary $R$-modules.

DEFINITION 2.1. A proper submodule $K$ of $M$ is called prime if $r m \in K$, for $r \in R$ and $m \in M$, then $r \in(K: M)$ or $m \in K$, where $(K: M)=\{r \in R \mid r M \subseteq$ $M\}$.

THEOREM 2.2 (see [5]). Let $K$ be a submodule of $M$. Then the following statements are satisfied:

(i) $K$ is prime if and only if $P=(K: M)$ is a prime ideal of $R$ and $R / P$-module $M / K$ is torsion-free,

(ii) if $(K: M)$ is a maximal ideal of $R$, then $K$ is a prime submodule of $M$.

For any $R$-module $M$, let $\operatorname{Spec}(M)$ denote the collection of all prime submodules of $M$. Note that some modules $M$ have no prime submodules (i.e., $\operatorname{Spec}(M)$ 
is empty); such modules are called primeless. For example, the zero-module is primeless. In [5], some nontrivial examples are shown and some conditions for primeless modules are given.

DEFINITION 2.3. An $R$-module $M$ is a multiplication module if for every submodule $N$ of $M$, there is an ideal $I$ of $R$ such that $N=I M$.

LEMMA 2.4 (see [1]). Let $M$ be a multiplication module and let $N$ be a submodule of $M$. Then $N=(\operatorname{ann}(M / N)) M$.

LEMMA 2.5 (see [1, Proposition 1.1]). An R-module $M$ is a multiplication if and only if for each $m$ in $M$, there exists an ideal $I$ of $R$ such that $R m=I M$.

LEMMA 2.6 (see [1]). An $R$-module $M$ is a multiplication if and only if

$$
\cap_{\lambda \in \Lambda}\left(I_{\lambda} M\right)=\left(\cap_{\lambda \in \Lambda}\left[I_{\lambda}+\operatorname{ann}(M)\right]\right) M
$$

for any collection of ideals $I_{\lambda}(\lambda \in \Lambda)$ of $R$.

Theorem 2.7 (see [1, Theorem 2.5]). Let $M$ be a nonzero multiplication $R$ module. Then,

(i) every proper submodule of $M$ is contained in a maximal submodule of $M$;

(ii) $K$ is a maximal submodule of $M$ if and only if there exists a maximal ideal $P$ of $R$ such that $K=P M \neq M$.

THEOREM 2.8 (see [1, Corollary 2.11]). The following statements are equivalent for a proper submodule $N$ of $M$ :

(i) $N$ is a prime submodule of $M$;

(ii) $\operatorname{ann}(M / N)$ is a prime ideal of $R$;

(iii) $N=P M$ for some prime ideal $P$ of $R$ with $\operatorname{ann}(M) \subseteq P$.

THEOREM 2.9 (see [1, Theorem 3.1]). Let $M$ be a faithful multiplication $R$ module. Then the following statements are equivalent:

(i) $M$ is finitely generated;

(ii) $A M \subseteq B M$ if and only if $A \subseteq B$;

(iii) for each submodule $N$ of $M$, there exists a unique ideal I of $R$ such that $N=I M$;

(iv) $M \neq A M$ for any proper ideal $A$ of $R$;

(v) $M \neq P M$ for any maximal ideal $P$ of $R$.

DEFINITION 2.10. Let $N$ be a proper submodule of $M$. Then, the radical of $N$ denoted by $M-\operatorname{rad}(N)$ or $r(N)$ is defined in [1] to be the intersection of all prime submodules of $M$ containing $N$.

THEOREM 2.11 (see [1, Corollary 2.11]). Let $N$ be a proper submodule of a multiplication $R$-module $M$. Then $M-\operatorname{rad}(N)=\sqrt{A} M$, where $A=\operatorname{ann}(M / N)$. 
Definition 2.12. Let $M$ be an $R$-module. Then, the radical of $M$ denoted by $\operatorname{rad}(M)$ is defined to be the intersection of the maximal submodules of $M$ if such exists, and $M$ otherwise.

Let $M$ denote the collection of all maximal ideals of $R$. Define $P_{1}(M)=\{P \in$ $\mathcal{M} \mid M \neq P M\}$ and $P_{2}(M)=\{P \in \mathcal{M} \mid \operatorname{ann}(M) \subseteq P\}$. Now, define $J_{1}(M)=\cap\{P \mid$ $\left.P \in P_{1}(M)\right\}$ and $J_{2}(M)=\cap\left\{P \mid P \in P_{2}(M)\right\}$.

THEOREM 2.13 (see [1, Theorem 2.7]). Let $M$ be a multiplication R-module. Then $\operatorname{rad}(M)=J_{1}(M) M=J_{2}(M) M$.

\section{The product of multiplication submodules}

DEFINITION 3.1. Let $M$ be an $R$-module and let $N$ be a submodule of $M$ such that $N=I M$ for some ideal $I$ of $R$. Then, we say that $I$ is a presentation ideal of $N$ or, for short, a presentation of $N$. We denote the set of all presentation ideals of $N$ by $\operatorname{Pr}(N)$.

Note that it is possible that for a submodule $N$, no such presentation ideal exists. For example, if $V$ is a vector space over an arbitrary field with a proper subspace $W(\neq 0$ and $V$ ), then $W$ does not have any presentations. By Lemma 2.4 , it is clear that every submodule of $M$ has a presentation ideal if and only if $M$ is a multiplication module. In particular, for every submodule $N$ of a multiplication module $M$, ann $(M / N)$ is a presentation for $N$.

Let $L(R)$ and $L(M)$ denote the lattices of ideals of $R$ and submodules of $M$, respectively. Define the relation $\sim$ on $L(R)$ as follows:

$$
I \sim J \Longleftrightarrow I M=J M .
$$

It is easy to verify that this relation is an equivalence relation on $L(R)$. We denote the equivalence class of $I \in L(R)$ by $[I]$.

THEOREM 3.2. Let $M$ be a faithful multiplication $R$-module. Then, the following statements are equivalent:

(i) $M$ is finitely generated;

(ii) each equivalence class of the relation $\sim$ is a singleton;

(iii) the map

$$
\varphi: L(R) \longrightarrow L(M)
$$

defined by $\varphi(I)=I M$ is a lattice isomorphism;

(iv) for every proper ideal I of $R,[I]=\{I\}$;

(v) for any maximal ideal $P$ of $R,[P]=\{P\}$.

Proof. (i) $\Rightarrow$ (ii) follows from Theorem 2.8, Definition 3.1, and Theorem 2.9. (ii) $\Rightarrow$ (iii). By Theorem 2.8, we conclude that $\varphi$ is bijective and order-preserving. Obviously, $(I+J) M=I M+J M$ and by Lemma 2.5, $(I \cap J) M=I M \cap J M$ since $M$ is faithful. Therefore, $\phi$ is a lattice isomorphism. 
(iii) $\Rightarrow$ (iv), (iv) $\Rightarrow$ (v), and (v) $\Rightarrow$ (i) are an immediate consequence of Theorem 2.8.

DEFINITION 3.3. Let $N=I M$ and $K=J M$ for some ideals $I$ and $J$ of $R$. The product of $N$ and $K$ is denoted by $N \cdot K$ or $N K$ is defined by $I J M$.

Clearly, $N K$ is a submodule of $M$ and contained in $N \cap K$. Now, we show that the product of two submodules is defining an operation on submodules of $M$.

THEOREM 3.4. Let $N=I M$ and $K=J M$ be submodules of a multiplication $R$-module $M$. Then, the product of $N$ and $M$ is independent of presentations of $N$ and $K$.

Proof. Let $N=I_{1} M=I_{2} M=N^{\prime}$ and $K=J_{1} M=J_{2} M=K^{\prime}$ for ideals $I_{i}$ and $J_{i}$ of $R, i=1$,2. Consider $r s m \in N K=I_{1} J_{1} M$ for some $r \in I_{1}, s \in J_{1}$, and $m \in M$. From $J_{1} M=J_{2} M$, we have

$$
s m=\sum_{i=1}^{n} r_{i} m_{i}, \quad r_{i} \in J_{2}, m_{i} \in M .
$$

Then,

$$
r s m=\sum_{i=1}^{n} r_{i}\left(r m_{i}\right)
$$

From $r m_{i} \in I_{1} M=I_{2} M$, we conclude that

$$
r m_{i}=\sum_{j=1}^{k} t_{i j} m_{i j}^{\prime}, \quad t_{i j} \in I_{2}, m_{i j}^{\prime} \in M .
$$

Thus,

$$
r s m=\sum_{i=1}^{n} \sum_{j=1}^{k} r_{i} t_{i j} m_{i j}^{\prime}
$$

Therefore, $r s m \in I_{2} J_{2} M$, and hence $I_{1} J_{1} M \subseteq I_{2} J_{2} M$. Similarly, we have $I_{2} J_{2} M \subseteq I_{1} J_{1} M$. This completes the proof.

Proposition 3.5. Let $M$ be a multiplication module $N$, and let $K$ and $L$ be submodules of $M$. Then the following statements are satisfied:

(i) $L(M)$, the lattice of submodules of $M$ with operation product on submodules, is a semiring;

(ii) the product is distributive with respect to the sum on $L(M)$;

(iii) $(K+L)(K \cap L) \subseteq K L$;

(iv) $K \cap L=K L$ provided $K+L=M$ (in this case, $K$ and $L$ are said to be coprime or comaximal). 
Proof. (i), (ii), (iii) are obtained from Definition 3.3, Lemma 2.5, the wellknown related results of the ideals theory, and the fact that $\sum_{k \in K} I_{k} M=$ $\left(\sum_{k \in K} I_{k}\right) M$.

(iv) $K+L=M$ implies that $M(K \cap L) \subseteq K L$ by (iii), and hence $K \cap L \subseteq K L$. Clearly $K L \subseteq K \cap L$. Therefore $K L=K \cap L$.

LEMMA 3.6. Let $N$ and $K$ be submodules of a multiplication module $M$. Then,

(i) the ideals $\operatorname{ann}(M / N) \cdot \operatorname{ann}(M / K)$ and $\operatorname{ann}(M / N K)$ are presentations of $N K$;

(ii) if $M$ is finitely generated, then $\operatorname{ann}(M / N) \cdot \operatorname{ann}(M / K)=\operatorname{ann}(M / N K)$.

Proof. (i) By Lemma 2.4 and Theorem 3.4, $\operatorname{ann}(M / N)$ and $\operatorname{ann}(M / K)$ are presentations for $N$ and $K$, respectively. Thus, by Definition 3.3, $M N=$ $[\operatorname{ann}(M / N) \cdot \operatorname{ann}(M / K)] M$. Therefore, $(\operatorname{ann}(M / N) \cdot \operatorname{ann}(M / K))$ is a presentation for $M N$.

(ii) By Lemma 2.4, we have $M N=\operatorname{ann}(M / N K)$ and hence by Theorem 2.8 and (i), we conclude that

$$
\operatorname{ann}(M / N) \cdot \operatorname{ann}(M / K)=\operatorname{ann}(M / N K) .
$$

REMARK 3.7. (i) Recall that by Lemma 2.5 , for any $m \in M$, we have $R m=I M$ for some ideal $I$ of $R$. In this case, we say that $I$ is a presentation ideal of $m$ or, for short, a presentation of $m$ and denote it by $\operatorname{Pr}(m)$. In fact, $\operatorname{Pr}(m)$ is equal to $\operatorname{Pr}(R m)$.

(ii) For $m, m^{\prime} \in M$, by $m m^{\prime}$, we mean the product of $R m$ and $R m^{\prime}$, which is equal to $I J M$ for every presentation ideals $I$ and $J$ of $m$ and $m^{\prime}$, respectively.

Proposition 3.8. Let $M$ be a multiplication $R$-module. Let $N, K, N_{i} \in I$ be submodules of $M, s \in R$, and $k$ any positive integer. Then the following statements are satisfied:

(i) $\operatorname{Pr}\left(\sum_{i \in I} N_{i}\right)=\sum_{i \in I} \operatorname{Pr}\left(N_{i}\right)$;

(ii) $\operatorname{Pr}\left(\cap_{i \in I} N_{i}\right)=\left(\cap_{i \in I}\left[\operatorname{Pr}\left(N_{i}\right)+\operatorname{ann}(M)\right]\right) M$;

(iii) $\operatorname{Pr}\left(\sum_{i=1}^{k} m_{i}\right) \subseteq \sum_{i=1}^{k} \operatorname{Pr}\left(m_{i}\right)$;

(iv) $\operatorname{Pr}(s m)=s \operatorname{Pr}(m)$;

(v) $\operatorname{Pr}(N K)=\operatorname{Pr}(N) \cdot \operatorname{Pr}(K)$;

(vi) $\operatorname{Pr}\left(N^{k}\right)=(\operatorname{Pr}(N))^{k}$;

(vii) $\operatorname{Pr}\left(m^{k}\right)=(\operatorname{Pr}(m))^{k}$;

(viii) $\operatorname{Pr}(M-\operatorname{rad}(N))=M-\operatorname{rad}(\operatorname{Pr}(N))$.

Proof. (i) Let $I_{i}$ be presentation ideals of $N_{i}$ for every $i \in I$. Then it is easy to verify that

$$
\sum_{i \in I} N_{i}=\sum_{i \in I}\left(M_{i}\right)=\left(\sum_{i \in I} I_{i}\right) M .
$$

Thus, $\operatorname{Pr}\left(\sum_{i \in I} N_{i}\right)=\sum_{i \in I} \operatorname{Pr}\left(N_{i}\right)$. 
(ii) It is an immediate consequence of Lemma 2.6.

(iii) By Remark 3.7(i), we have

$$
\operatorname{Pr}\left(\sum_{i=1}^{k} m_{i}\right)=\operatorname{Pr}\left(R \sum_{i=1}^{k} m_{i}\right) \subseteq \operatorname{Pr}\left(R \sum_{i=1}^{k} R m_{i}\right)=\operatorname{Pr}\left(\sum_{i=1}^{k} R m_{i}\right)=\sum_{i=1}^{k} \operatorname{Pr}\left(m_{i}\right) .
$$

(iv), (v), (vi), and (vii) are an immediate consequence of Theorem 3.4 and Remark 3.7.

(viii) It follows from Theorem 2.11.

Definition 3.9. Let $M$ be a multiplication $R$-module and let $N$ be a submodule of $M$. Then,

(i) $N$ is called nilpotent if $N^{k}=0$ for some positive integer $k$, where $N^{k}$ means the product of $N, k$ times;

(ii) an element $m$ of $M$ is called nilpotent if $m^{k}=0$ for some positive integer $k$.

The set of all nilpotent elements of $M$ is denoted by $N_{M}$.

THEOREM 3.10. Let $M$ be a multiplication module. A submodule $N$ of $M$ is nilpotent if and only if for every presentation ideal I of $N, I^{k} \subseteq \operatorname{ann}(M)$ for some positive integer $k \in \mathbb{N}$.

Proof. Let $I$ be a presentation ideal of $N$. If $N$ is nilpotent, then $N^{k}=0$ for some positive integer $k$, that is, $N^{k}=I^{k} M=0$. Thus, $I^{k} \subseteq$ ann $(M)$. Conversely, suppose that $I^{k} \subseteq \operatorname{ann}(M)$ for some presentation ideal $I$ of $N$. Then,

$$
N^{k}=I^{k} M \subseteq \operatorname{ann}(M) M=0 .
$$

Therefore, $N$ is nilpotent.

COROLlary 3.11. Let $M$ be a faithful $R$-multiplication module and let $N$ be a submodule of $M$. Then, $N$ is nilpotent if and only if every presentation ideal of $N$ is a nilpotent ideal.

THEOREM 3.12. Let $M$ be a multiplication module. Then, $N_{M}$ is a submodule of $M$ and $M / N_{M}$ has no nonzero nilpotent element.

Proof. Let $x, y \in N_{M}$, say $x^{m}=0$ and $y^{n}=0$. Consider presentation ideals $I$ and $J$ of $x$ and $y$, respectively. Then $x^{m}=I^{m} M=0$ and $y^{m}=I^{n} M=0$. Since $R x=I M$ and $R y=J M$, then by Lemma 2.5, we have $R(x+y) \subseteq R x+R y=$ $I M+J M=(I+J) M$, then $I+J$ is a presentation ideal for $x+y$. Let $l=m+n$. Then,

$$
(x+y)^{m+n}=(I+J)^{m+n} M=\left(\sum_{i=0}^{l}\left(\begin{array}{l}
l \\
i
\end{array}\right)(I)^{i}(J)^{l-i}\right) M=(0) M=(0),
$$


and hence $x+y \in N_{M}$. Now, let $m \in N_{M}$ and $r \in R$. Consider presentation ideal $I$ of $m$. Thus, $m^{k}=I^{k} M=0$ since $R r m=(r I) M \subseteq I M$. Thus, $(r m)^{k}=$ $(r I)^{k} M \subseteq I^{k} M=(0)$ and hence $r m \in N_{M}$. Therefore, $N_{M}$ is a submodule of $M$.

Let $\bar{x} \in M / N_{M}$ be represented by $x$. Then, $\overline{x^{n}}$ is represented by $x^{n}$ so that $\overline{x^{n}}=0$. Thus, $x^{n} \in N_{M}$ and hence $\left(x^{n}\right)^{k}=0$ for some $k \geq 0$. Therefore, $x \in N_{M}$ and so $\bar{x}=0$.

THEOREM 3.13. Let $N$ be a submodule of a multiplication $R$-module $M$. Then $M-\operatorname{rad}(N)=\left\{m \in M \mid m^{k} \subseteq N\right.$ for some $\left.k \geq 0\right\}$.

Proof. Let

$$
B=\left\{m \in M \mid m^{k} \subseteq N \text { for some } k \geq 0\right\} .
$$

First, we show that $B$ is a submodule of $M$. Let $x, y \in B$, and let $I$ and $J$ be presentation ideals of $x$ and $y$, respectively. Then, $x^{n}=I^{n}$ and $y^{m}=J M \subseteq N$ for some positive integers $m$ and $n$, and presentation ideals $I, J$ of $x$ and $y$, respectively. Let $k=\max \{m, n\}$. Then

$$
\begin{aligned}
(x+y)^{k} & =(I M+J M)^{k}=((I+J) M)^{k} \\
& =(I+J)^{k} M=\sum_{i=0}^{k}\left(\begin{array}{l}
k \\
i
\end{array}\right)(I M)^{i}(J M)^{k-i},
\end{aligned}
$$

that is, $x+y \in B$. Also, for $x \in B$ and $r \in R$, we have $(r x)^{n} \subseteq N$ since $x^{n} \subseteq N$. Thus, $B$ is a submodule of $M$. Suppose that $m \in B$ and $A$ is a presentation of $m$. Then, $m^{k}=A^{k} M \subseteq N$ for some $n \geq 1$ and hence by Theorem 2.11, we have

$$
M-\operatorname{rad}\left(m^{k}\right)=\sqrt{A^{k} M}=\sqrt{A} M \subseteq M-\operatorname{rad}(N) .
$$

Thus, $M-\operatorname{rad}(R m)=M-\operatorname{rad}(A M) \subseteq M-\operatorname{rad}(N)$ and this implies that $B \subseteq$ $M-\operatorname{rad}(N)$.

Conversely, let $m \in M-\operatorname{rad}(N)=\sqrt{I} M$, where $I=\operatorname{ann}(M / N)$. Then, $m=$ $\sum_{i=1}^{n} r_{i} m_{i}$ for $r_{i} \in \sqrt{I}$ and $m_{i} \in M$. Thus, $r_{i}^{n_{i}} \in I$ for some $n_{i} \geq 1$. Thus, for a sufficiently large $n$, we have $m^{k} \subseteq I M=N$ and hence $M-\operatorname{rad}(N) \subseteq B$. Therefore, $B=M-\operatorname{rad}(N)$.

COROLlARY 3.14. Let $M$ be a multiplication $R$-module. Then $N_{M}$ is the intersection of all prime submodules of $M$.

Proof. By Theorem 2.11, we have $M-\operatorname{rad}(0)=\sqrt{A} M$, where $A=\operatorname{ann}(M)$, and by Theorem 3.13, $M-\operatorname{rad}(N)=N_{M}$.

COROLlarY 3.15. Let $M$ be a faithful multiplication $R$-module. Then $N_{M}=$ $\mathcal{N} M$, where $\mathcal{N}$ is the nilradical of $R$. 
THEOREM 3.16. Let $P$ be a proper submodule of a multiplication module $M$. Then $P$ is prime if and only if

$$
U V \subseteq P \Longrightarrow U \subseteq P \quad \text { or } \quad V \subseteq P
$$

for each submodule $U$ and $V$ of $M$.

Proof. Let $P$ be prime and $U V \subseteq P$, but $U \nsubseteq P$ and $V \nsubseteq P$ for some submodules $U$ and $V$ of $M$. Suppose that $I$ and $J$ are presentations of $U$ and $V$, respectively. Then $U V=I J M \subseteq P$. Thus, there are $r y \in U-P$ and $s x \in U-P$ for some $r \in I$ and $s \in J$. Thus, $r s x \in P$ and hence $r M \subseteq P$, that is, $r y \in P$, which is a contradiction.

Conversely, suppose that condition (3.15) is true. Let $r x \in P$ for some $r \in R$ and $x \in M-P$, but $r M \nsubseteq P$; then, $r m \notin P$ for some $m \in M$. Let $I$ and $J$ be presentation ideals of $r x$ and $m$, respectively. Then

$$
R(r x) \cdot(R m)=(R x) \cdot(R r m)=I M \cdot J M=I J M \subseteq P .
$$

Now, by hypothesis, we must have $R x \subseteq P$ or $R r m \subseteq P$, which implies that $x \in P$ or $r m \in P$, which is a contradiction. Therefore, $P$ is prime.

COROLlaRY 3.17. Let $P$ be a proper submodule of $M$. Then $P$ is prime if and only if

$$
m \cdot m^{\prime} \subseteq P \Longrightarrow m \in P \quad \text { or } \quad m^{\prime} \in P
$$

for every $m, m^{\prime} \in M$.

Proof. If $P$ is prime, then, clearly, (3.17) is true. Conversely, suppose that (3.17) is true, and $U V \subseteq P$ for submodules $U$ and $V$ of $M$, but $U \nsubseteq P$ and $V \nsubseteq P$. Thus, there are $u \in U-P$ and $v \in V-P$. Then $u v=R u R v \subseteq U V \subseteq P$ and hence by (3.17), we must have $u \in U$ or $v \in V$, which is a contradiction. Therefore, $P$ is prime.

Definition 3.18. An element $u$ of an $R$-module $M$ is said to be a unit provided that $u$ is not contained in any maximal submodule of $M$.

THEOREM 3.19. Let $M$ be a multiplication $R$-module. Then $u \in M$ is a unit if and only if $\langle u\rangle=M$.

Proof. The sufficiency is clear. For a necessary part, let $u$ be a unit element. Then $\langle u\rangle$ is not contained in any maximal submodule of $M$. Thus, by Theorem 2.7, we must have $\langle u\rangle=M$.

THEOREM 3.20. Let $M$ be an $R$-module (not necessarily multiplicative) such that $M$ has a unit $u$. Then $m \in \operatorname{rad}(M)$ if and only if $u-r m$ is unit for every $r \in R$.

Proof. See [7, Theorem 4.8]. 
THEOREM 3.21. Every homomorphic image of a multiplication module is a multiplication module.

Proof. Let $M$ be a multiplication $R$-module, $\phi: M \rightarrow M^{\prime}$ an $R$-module homomorphism, and $K=\phi(M)$. Let $k \in K$, then $k=(\phi m)$ for some $m \in M$. Since $M$ is a multiplication, then by Lemma 2.5, there is an ideal $I$ of $R$ such that $R m=I M$. Thus,

$$
\varphi(I M)=I \varphi(M)=I K=\varphi(R m)=R \varphi(m)=R k .
$$

Therefore, by Lemma $2.5, K$ is a multiplication $R$-module.

COROLLARY 3.22. Let $M$ be a multiplication $R$-module and $N$ a submodule of $M$. Then, $M / N$ is a multiplication $R$-module.

THEOREM 3.23 (a version of Nakayama lemma). Let $M$ be a faithful multiplication $R$-module such that $M$ has a unit $u$. Then, for every submodule $N$, the following conditions are equivalent:

(i) $N$ is contained in every maximal submodule of $M$;

(ii) $u-r x$ is a unit for all $r \in R$ and for all $x \in N$;

(iii) if $M$ is a finitely generated $R$-module such that $N M=M$, then $M=0$;

(iv) if $M$ is finitely generated and $K$ is a submodule of $M$ such that $M=$ $N M+K$, then $M=K$.

Proof. (i) $\Rightarrow$ (ii) is an immediate consequence of Theorem 3.19.

(ii) $\Rightarrow$ (iii). Since $M$ is finitely generated, there must be a minimal generating set $X=\left\{m_{1}, \ldots, m_{n}\right\}$ of $M$. If $M \neq 0$, then $m_{1} \neq 0$ by minimality. Now, let $I$ be a presentation of $N$. Then, $N M=M$ implies that $M=I M \cdot M=M$, and since $M$ is faithful, then by Theorem 2.13, we have $N \subseteq \operatorname{rad}(M)=J_{1}(M) M \subseteq J(R) M$. Thus, $m_{1}=j_{1} m_{1}+j_{2} m_{2}+\cdots+j_{n} m_{n}\left(j_{i} \in J(R)\right)$ whence $j_{1} m_{1}=m_{1}$ so that $\left(1-j_{1}\right) m_{1}=0$ if $n=1$, and

$$
\left(1-j_{1}\right) m_{1}=j_{2} m_{2}+\cdots+j_{n} m_{n}, \quad n>1 .
$$

Since $1-j_{1}$ is a unit in $R, m_{1}=\left(1-j_{1}\right)^{-1}\left(1-j_{1}\right) m_{1}+\cdots+\left(1-j_{1}\right)^{-1} j_{n} m_{n}$. Thus, if $n=1$, then $m_{1}=0$, which is a contradiction. If $n>1$, then $m_{1}$ is a linear combination of $m_{2}, m_{3}, \ldots, m_{n}$; consequently, $\left\{m_{2}, \ldots, m_{n}\right\}$ generates $M$, which contradicts the choice of $X$.

(iii) $\Rightarrow$ (iv). Since for every submodule $K / N$ of $M / N$, we have $K / N=$ $\operatorname{ann}(M / N / K / N) M / N=\operatorname{ann}(M / K) M / N$; then by Corollary 3.22, $M / N$ is a multiplication $R$-module. Now, it is easy to verify that $\operatorname{rad}(M / N)=M / N$ and hence, by (iii), we must have $M=K$.

(iv) $\Rightarrow$ (i). Let $K$ be any maximal submodule of $M$, then $K \subseteq N M=K$. Consequently, $N M+M=M$ by maximality of $K$, otherwise $M=K$ by (iv) a contradiction. Therefore, $N=N M \subseteq K$. 


\section{REFERENCES}

[1] Z. Abd El-Bast and P. F. Smith, Multiplication modules, Comm. Algebra 16 (1988), no. 4, 755-779.

[2] A. Barnard, Multiplication modules, J. Algebra 71 (1981), no. 1, 174-178.

[3] J. Jenkins and P. F. Smith, On the prime radical of a module over a commutative ring, Comm. Algebra 20 (1992), no. 12, 3593-3602.

[4] C.-P. Lu, Prime submodules of modules, Comment. Math. Univ. St. Paul. 33 (1984), no. 1, 61-69.

[5] R. L. McCasland, M. E. Moore, and P. F. Smith, On the spectrum of a module over a commutative ring, Comm. Algebra 25 (1997), no. 1, 79-103.

[6] S. Singh and F. Mehdi, Multiplication modules, Canad. Math. Bull. 21 (1969), 10571061.

[7] M. M. Zahedi and R. Ameri, On the prime, primary and maximal subhypermodules, Ital. J. Pure Appl. Math. (1999), no. 5, 61-80.

Reza Ameri: Mathematics Department, Faculty of Basic Sciences, University of Mazandaran, Babolsar, Iran

E-mail address: ameri@umz.ac.ir 


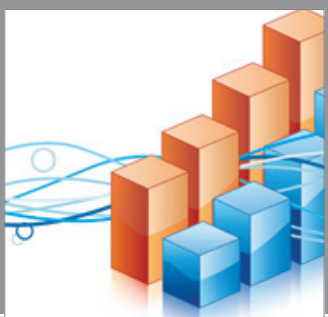

Advances in

Operations Research

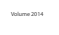

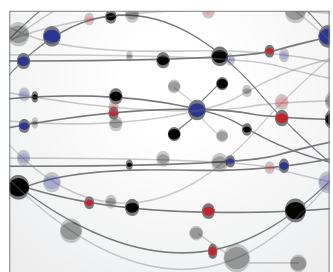

\section{The Scientific} World Journal
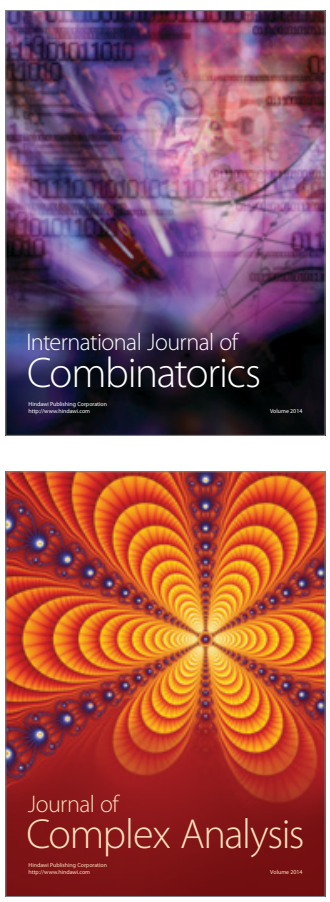

International Journal of

Mathematics and

Mathematical

Sciences
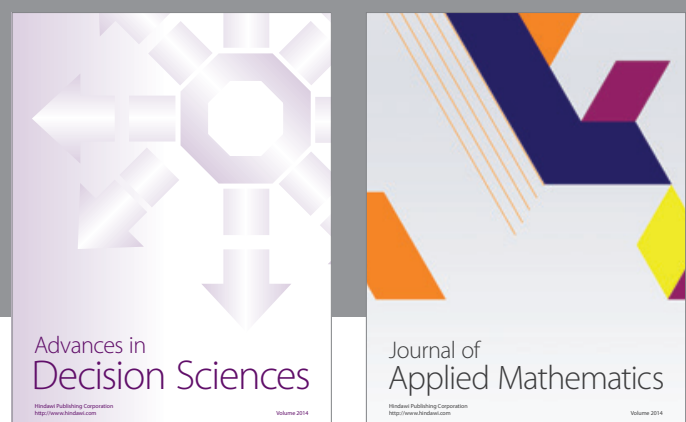

Journal of

Applied Mathematics
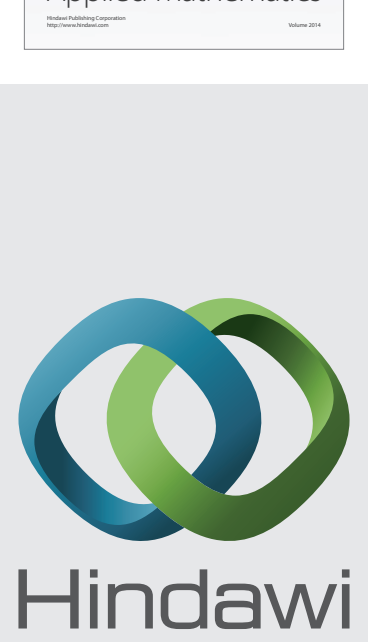

Submit your manuscripts at http://www.hindawi.com
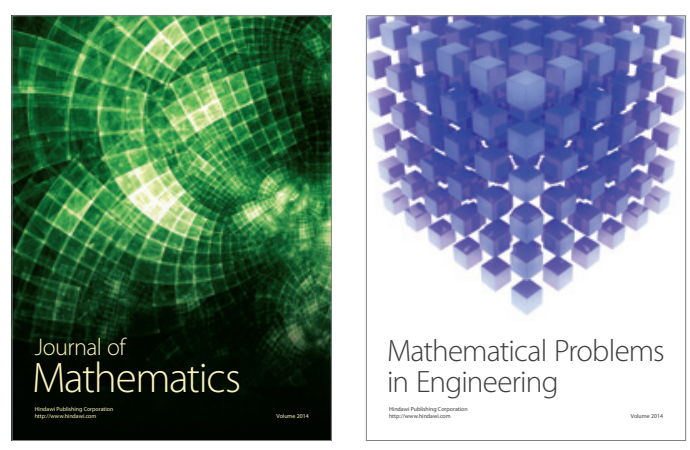

Mathematical Problems in Engineering
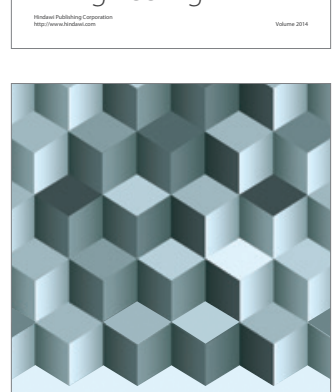

Journal of

Function Spaces
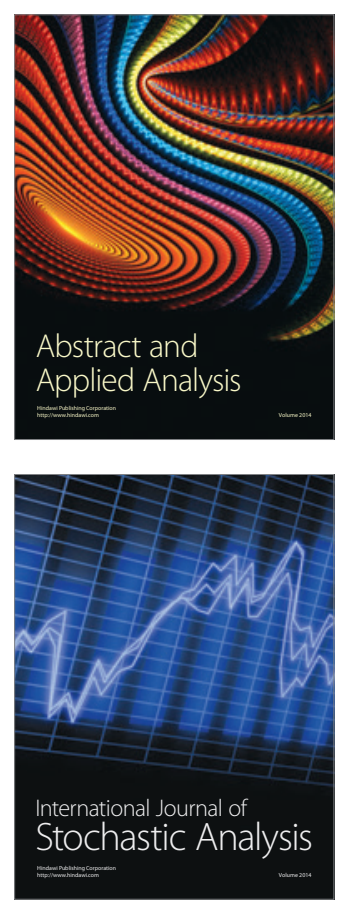

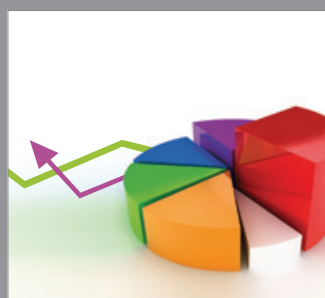

ournal of

Probability and Statistics

Promensencen
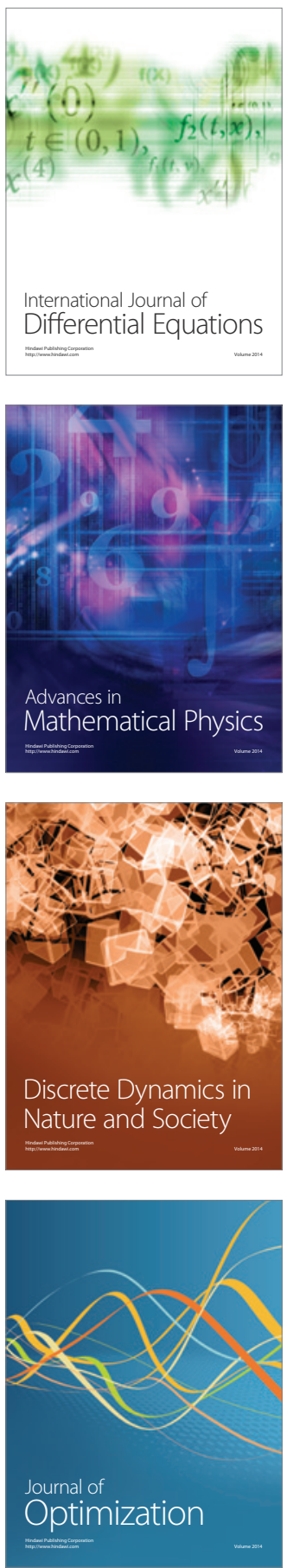